\title{
Photospheric data programs at the Debrecen Observatory
}

\author{
L. Győri, T. Baranyi, and A. Ludmány \\ Heliophysical Observatory of the Hungarian Academy of Sciences, \\ 4010 Debrecen, P.O. Box 30, Hungary \\ email: gylajos@tigris.unideb.hu, baranyi@tigris.unideb.hu, \\ ludmany@tigris.unideb.hu
}

\begin{abstract}
The primary task of the Debrecen Observatory is the most detailed, reliable and precise documentation of the solar photospheric activity. This long-term effort started with the continuation of the Greenwich photoheliograph program, this is the Debrecen Photoheliographic Data (DPD) sunspot catalogue based on ground-based observations. The profile of the work has later been extended to space-borne observations (SOHO/MDI and SDO), to magnetic fields and faculae as well as to higher temporal resolution (one hour) and nearly real-time data supply. The database also includes historical observations. The web-presentation developed for the material is easy to search and browse. We describe the main characteristics of these catalogs, and their advantages. We summarize the recent advances in the procedure of their compilation, and the available sets of the data and images.
\end{abstract}

Keywords. Sun: sunspots, faculae, activity, photosphere, magnetic fields

\section{Debrecen Photoheliographic Data}

Position and area data of sunspot groups for every day were published at Greenwich (Greenwich Photoheliographic Results, GPR) until 1976. After that, Debrecen Heliophysical Observatory took over this responsibility. The Debrecen Photoheliographic Data (DPD) is mainly compiled by using white light full disk observations taken at Debrecen Observatory and its Gyula Observing Station with an archive containing more than 150,000 photoheliograms observed since 1958. Observations of several other observatories help in making the catalogue complete.

The final version of DPD completely covers the whole year with one observation/day time resolution similar to the GPR, and it contains the same data for the sunspot groups. In addition, it gives account of all sunspots, even the smallest observable ones, both umbrae and penumbrae. The estimated mean precision of position data is $\sim 0.1$ heliographic degrees, and that of area data is $\sim 10$ percents.

The instrumental background for DPD catalogue compilation has changed over the years. Recently a professional scanner is used to obtain full disk scans of photoheliograms on films or glass plates with $8 \mathrm{kx} 8 \mathrm{k}$ spatial resolution to avoid any loss of information. Recently a 4kx4k CCD camera is being used for the observations in the Gyula Observing Station. In addition, several $2 \mathrm{kx} 2 \mathrm{k}$ or $1 \mathrm{kx} 1 \mathrm{k}$ CCD observations of different observatories are measured for the DPD. The rate of space-borne observations applied for DPD has also increased recently. The quality of the available films is decreasing with the increasing usage of CCD cameras, but the spatial resolution of CCD cameras has not yet reached the resolution of the former high quality films. Thus, a large amount of SOHO/MDI images were measured for DPD in the recent years to provide data of the best available quality. It is probable that this trend will continue in the next years by using the SDO images of the highest quality. However, the ground-based observations remain essential 
to contribute to the completeness of the material, data validation, comparison, and the security of data flow.

The software package called SAM (Sunspot Automatic Measurement) (Györi 1998, 2003, 2005) was developed to handle the scans of the ground-based photographic observations. After that, it was further developed to handle the ground-based and space-borne CCD FITS images. Now it can be applied to any full disk digitized solar image if the orientation of the image is known or can be derived from the image itself. The automatic sunspot-measuring program determines the borders of umbrae and penumbrae, finds the centers of spots. The package automates (as far as possible) the compilation of the sunspot catalogue which contains all relevant pieces of information about the spots.

The DPD is available for 1986-2010 at present. For some years, the final version is published but for other years only a preliminary version is available. However, the DPD is continuously improved, revised, and extended in its content. The efforts to achieve the full coverage of the post GPR-era also continue. In addition, we have started compiling catalogs of higher-level data (e.g. catalogue of sunspot group tilt angles, catalogue of recurrent active regions).

\section{SOHO/MDI-Debrecen Data}

The SOHO/MDI images (1024x1024 pixel) obtained as proxies for the continuum intensity near the NiI absorption line at $676.8 \mathrm{~nm}$ by combining the standard five filtergrams (Scherrer at al. 1995). Recently the compilation of SOHO/MDI-Debrecen Data (SDD) hourly sunspot catalogue has been started by using the SOHO/MDI continuum intensity images and magnetograms. This catalogue is similar to that of DPD in its data format and image products but the time cadence is 1 hour when MDI observations allow. The other difference is that the SDD contains magnetic information because the SAM is suitably modified to determine the mean line-of-site magnetic field of umbral and penumbral parts of spots from the quasi-simultaneous magnetogram.

We use the Full Disk Continuum images from the Hourly Data Sets Level 1.8 and the recently recalibrated magnetograms. In this data set, there are observations with different time resolution (from one/day to one/ $\mathrm{min}$ ) in different time intervals. In the latest years, usually there is one intensity image per hour but not in every hour. To get a data set with a more or less regular time cadence we decided to use roughly one image/hour time resolution. The best available intensity image is chosen within the time interval (hour - $30 \mathrm{~min}$, hour $+30 \mathrm{~min}$ ) which is the closest to the center of the time interval and the time-difference between the next images is at least 30 minutes. The best available magnetograms closest in time to the intensity images are chosen from the merged data sets of Full Disk Magnetograms of the Hourly Data Sets and Daily Data Sets. The set of images contains the intensity images with the nearest magnetograms selected after using quality-filters and time sequence criteria. If an hourly data is missing from the catalogue it means that there is no intensity observation within the given time interval, or the available intensity observation is not measurable (perfect), or there are no measurable intensity images and magnetograms available with less than $50 \mathrm{~min}$ time difference. The full disk SOHO/MDI intensity images are enlarged (3x), corrected for limb darkening, flat fielded and transformed to negative. If the position angle of the image is 180 degree, the processed image is rotated to the normal position; otherwise, the image preserves its original orientation.

The full-disk version of the SDD catalogue (fdSDD) is based on the same hourly time resolution MDI observations, but the spots are numbered by the computer program on 
the basis of their longitude and they is not assigned to sunspot groups. In the fdSDD catalogue, all sunspots have their area, position and magnetic field values similar to SDD sunspot group catalog.

\section{Presentation of sunspot data}

The electronic versions of the DPD and SDD are freely downloadable and usable. Their formats and contents are developed continuously to provide an easily usable set of sunspot data and solar images. Starting from this year the dataset has been refreshed with the data for recent days on a daily basis.

Several kinds of numerical data are presented in ASCII files: 1. yearly tables for daily (DPD) and hourly (SDD) sums of area data 2. time series of daily data 3. tables containing the whole area of sunspot groups and their mean position data, 4 . tables for the sunspot area and position data, 5. combined datasets containing all data.

The appended images show the detailed morphology. Scans of sunspot groups are published in JPG and FITS format with numbered spots. Full disk white light images and magnetic observations are appended to provide the most information available concerning the sunspots. The processed white-light or continuum images are published on-line in about $4 \mathrm{kx} 4 \mathrm{k}$ or $3 \mathrm{kx} 3 \mathrm{k}$ image size. The magnetograms are available in its original format and converted to JPG images too.

These data and images are accessible by ftp to provide an easy bulk download. The whole material is also provided in a graphical presentation containing daily full-disk drawings of the observed sunspot groups, and the full-disk white-light and magnetic observations. The days can be surveyed by turning the pages, and all the information on a sunspot group can be reached by clicking on the group number.

A new facility has been developed recently. There is an on-line MySQL query at the website of the catalogues which makes possible a quick and easy selection of the numerical data.

\section{Catalogue of white-light faculae}

A further novelty at the Debrecen Observatory is the publication of a catalogue of continuum faculae. It is based on the SOHO/MDI full disc images for the whole SOHO era. The full-disk facular data have the same format as full-disk sunspot data, but umbral areas are obviously not measured for faculae, and the first column of magnetic data contains the line-of-site magnetic field value at the brightest pixel within the facular contour in the intensity image.

\section{Historical Solar Image Database (HSID)}

A long-term effort is in progress to reveal, gather, digitize and evaluate all existing historical full-disc solar observations. The Debrecen Observatory preserves historical drawings between 1872 and 1919 gathered by two observatories: the material of Ógyalla Observatory (founded by Miklós Konkoly-Thege, 1842-1916) covers the period 1872-1891, the observations of Haynald Observatory in Kalocsa are made by Julius Fényi S.J. between 1880 and 1919. The old observations are indispensable in the long-term studies of solar activity, and they contain an invaluable amount of information, which can and must be reconstructed with the recent technology. 

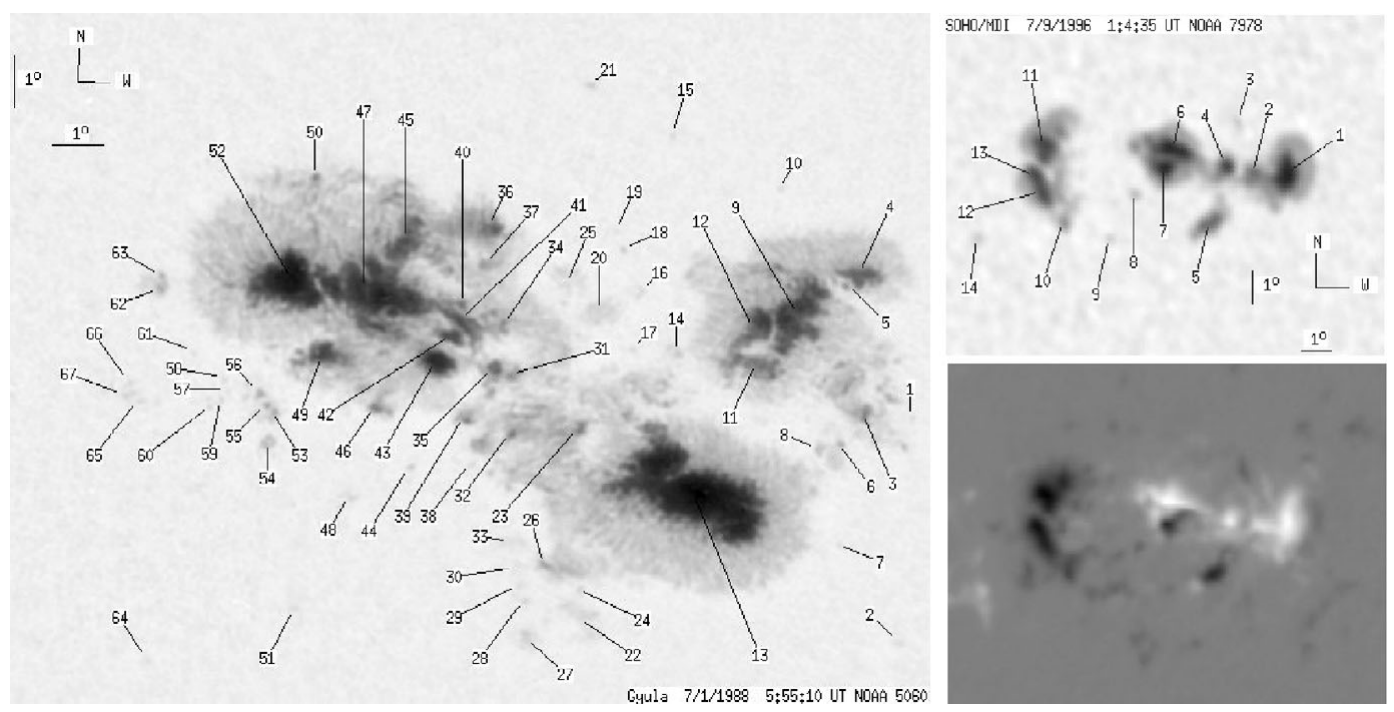

Figure 1. Example for an image of a sunspot group in DPD, and an image of a sunspot group from SDD with its magnetogram.

\section{Summary}

Both DPD and SDD contain data of all sunspot groups and sunspots, even those of the smallest observable ones. The estimated mean precision of position data is $\sim 0.1$ heliographic degrees, and that of area data is $\sim 10$ percents.

The DPD is mostly suitable for long-term studies because of the daily sampling, its spatial scale extends from full disc to about 1 arcsec. Additional data are also provided, e.g. catalogues of sunspot group tilts and recurrent active regions.

An additional advantage of the SDD catalogue is the roughly hourly time resolution, furthermore, it also contains magnetic information for the sunspots.

The content of the online database and its presentation: numerical datasets; appended images of active regions with numeration of spots (jpg, fits); full disc white-light or continuum images appended; magnetic observations appended; graphical HTML-presentation with full-disk and sunspot group images; on-line MySQL query for the numerical database; quick-look catalogue refreshed on a daily basis.

All the data and images mentioned in this paper are available at the site of Debrecen Observatory at http://fenyi.solarobs.unideb.hu/. Some further information can also be found here concerning the data, the observations, and the contributing observatories. The data sets are available without restrictions, but the users should refer to this paper in the References when the data or images are used in a scientific publication.

\section{Acknowledgements}

This work was supported by the European Community's Seventh Framework Program (FP7 SP1-Cooperation) under grant agreement No. 218816.

$\mathrm{SOHO}$ is a mission of international cooperation between ESA and NASA. The authors gratefully acknowledge the past and ongoing effort of the MDI team.

We express our deepest gratitude to the colleagues at the 16 collaborating observatories for putting the necessary material at our disposal. 


\section{References}

Győri, L. 1998, Solar Phys., 180, 109

Győri, L., 2003, Compiling sunspot catalogue: the principles, Proc. Solar Image Recognition Workshop (SIRW), Brussels, 2003

Győri, L., 2005, Automated determination of the alignment of solar images, Hvar Obs. Bull., 29, 299

Scherrer, P. H., Bogart, R. S., Bush, R. I., et al. 1995, Solar Phys., 162, 129 